\title{
ApoE Regulates the Development of Adult Newborn Hippocampal Neurons
}

\author{
Yacine Tensaouti, ${ }^{1}$ Elizabeth P. Stephanz, ${ }^{1}$ ํ)Tzong-Shiue Yu, ${ }^{1}$ and ${ }^{\mathbb{D}}$ Steven G. Kernie ${ }^{1,2}$ \\ DOI:http://dx.doi.org/10.1523/ENEURO.0155-18.2018 \\ ${ }^{1}$ Department of Pediatrics, Columbia University College of Physicians and Surgeons, New York, NY 10032 and \\ ${ }^{2}$ Department of Neurology, Columbia University College of Physicians and Surgeons, New York, NY 10032
}

\begin{abstract}
Adult hippocampal neurogenesis occurs throughout life and is believed to participate in cognitive functions such as learning and memory. A number of genes that regulate adult hippocampal neurogenesis have been identified, although most of these have been implicated in progenitor proliferation and survival, but not in the development into fully differentiated neurons. Among these genes, apolipoprotein $E(A p o E)$ is particularly compelling because the human ApoE isoform E4 is a risk factor for the development of Alzheimer's disease, where hippocampal neurogenesis is reported to be dysfunctional. To investigate the effects of ApoE and its human isoforms on adult hippocampal neurogenesis and neuronal development, retroviruses carrying a GFP-expressing vector were injected into wild-type (WT), ApoE-deficient, and human targeted replacement (ApoE3 and ApoE4) mice to infect progenitors in the dentate gyrus and analyze the morphology of fully developed GFP-expressing neurons. Analysis of these adult-born neurons revealed significant decreases in the complexity of dendritic arborizations and spine density in ApoE-deficient mice compared with WT mice, as well as in ApoE4 mice compared with ApoE3. These findings demonstrate that ApoE deficiency and the ApoE4 human isoform both impair hippocampal neurogenesis and give insight into how ApoE may influence hippocampal-related neurological diseases.
\end{abstract}

Key words: ApoE; dentate gyrus; neurogenesis

\section{Significance Statement}

Apolipoprotein $\mathrm{E}$ (ApoE) is known to regulate postnatal neurogenesis in the dentate gyrus of the hippocampus by directly affecting the proliferation of early progenitor cells. We found reduced complexity of adult-born granule cell dendritic arborizations as well as reduced spine density in ApoE-deficient and ApoE4 mice dentate gyrus neurons. These results provide strong evidence of impaired development of adult-born neurons in ApoE-deficient and ApoE4 mouse hippocampus, which may help to explain the higher risk of hippocampal-related neuropsychiatric diseases in humans carrying the E4 allele.

\section{Introduction}

Increasing evidence suggests that some cognitive deficits, difficulty in learning new information and memory loss, are due to alterations in adult dentate gyrus neurogenesis, a key component of hippocampus-associated neurologic diseases such as major depressive disorder

Received April 17, 2018; accepted June 25, 2018; First published July 30, 2018.

The authors declare no competing financial interests.

Author contributions: S.G.K. and T.-S.Y. designed research; Y.T. performed research; E.P.S. analyzed data; Y.T., E.P.S., T.-S.Y. , and S.G.K. wrote the paper.
(Sahay and Hen, 2007), schizophrenia (Allen et al., 2016), Alzheimer's disease (AD; Perry et al., 2012; Hollands et al., 2016), traumatic brain injuries (Hong et al., 2016), and epilepsy (Cho et al., 2015). Therefore, understanding the mechanisms underlying adult neurogenesis is essential before hippocampal progenitor manipulation can be a

This research was supported by National Institutes of Health/National Institute of Neurological Disorders and Stroke Grants R56-NS-089523 and R01NS-095803.

Correspondence should be addressed to either Tzong-Shiue Yu or Steven G. Kernie, Columbia University College of Physicians and Surgeons, New York, New York 10032, E-mail: ty2255@cumc.columbia.edu or sk3516@cumc.columbia.edu 
Table 1. Key resources table, highlighting the genetically modified organisms and strains, viruses and software essential to reproduce results presented in the manuscript

\begin{tabular}{|c|c|c|}
\hline $\begin{array}{l}\text { Reagent or resource } \\
\text { Bacterial and virus strains }\end{array}$ & Source & Identifier \\
\hline $\begin{array}{l}\text { Moloney murine leukemia viral vectors, } \\
\text { RV-CAG-eGFP or RV-CAG-eGFPcre }\end{array}$ & GT3 Core Facility of the Salk Institute & NIH-NCI CCSG: P30 014195, NINDS R24 Core Grant, NEI \\
\hline Experimental models: organisms/strains & & \\
\hline B6.129P2-Apoetm1Unc/J & The Jackson Laboratory & Catalog \#JAX:002052; RRID:IMSR_JAX:002052, https://www.jax.org/strain/002052 \\
\hline B6.129P2-Apoetm2(APOE*3)Mae N8 & Taconic & Catalog \#TAC:1548; RRID:IMSR_TAC:1548, https://www.taconic.com/transgenic-mouse-model/apoe3 \\
\hline B6.129P2-Apoetm3(APOE*4)Mae N8 & Taconic & Catalog \#TAC:1549; RRID:IMSR_TAC:1549, https://www.taconic.com/transgenic-mouse-model/apoe4 \\
\hline $\begin{array}{l}\text { B6;129S6-Gt(ROSA)26Sortm9(CAG- } \\
\text { tdTomato)Hze/J }\end{array}$ & The Jackson Laboratory & Catalog \#JAX: 007909; RRID:IMSR_JAX:007905, https://www.jax.org/strain/007905 \\
\hline C57BL/6J & The Jackson Laboratory & Catalog \#JAX:000664; RRID:IMSR_JAX:000664, https://www.jax.org/strain/000664 \\
\hline Software & & \\
\hline Adobe Photoshop & Adobe & RRID:SCR_014199, https://www.adobe.com/products/photoshop.html \\
\hline AutoQuant & Media Cybernetics & RRID:SCR_002465, http://www.mediacy.com/index.aspx?page=AutoQuant \\
\hline Prism & GraphPad Software & RRID:SCR_015807, https://www.graphpad.com/scientific-software/prism/ \\
\hline Neurolucida & mbf BIOSCIENCE & RRID:SCR_001775, http://www.mbfbioscience.com/neurolucida \\
\hline Stereo Investigator & mbf BIOSCIENCE & RRID:SCR_002526, http://www.mbfbioscience.com/stereo-investigator \\
\hline
\end{tabular}

RRID, Research resource identifiers (https://scicrunch.org/resources).

viable target in treating or alleviating symptoms of neurologic and neurodegenerative diseases.

Despite increasing knowledge regarding the developmental steps that control hippocampal neurogenesis and the integration of adult-born granule neurons in the preexisting circuitry, key regulatory genes underlying this process remain largely unknown. By comparing the expression profile of neural stem and progenitor cells (NSPCs) from mouse dentate gyrus at postnatal days 7 and 28, apolipoprotein E (ApoE) was identified as a potential regulator of adult neurogenesis (Gilley et al., 2011) and subsequently shown to regulate developmental and postnatal neurogenesis in the dentate gyrus by preventing overproliferation of NSPCs (Yang et al., 2011). In addition, ApoE deficiency is known to shift neural stem cell differentiation toward astrogenesis and away from neurogenesis (Li et al., 2009).

ApoE in the brain is produced and secreted primarily by astrocytes but is also expressed in type 1 neural stem cells (Hong et al., 2016). ApoE is involved in the regulation of lipid transport mostly from astrocytes to neurons, synaptogenesis, lipid intracellular homeostasis, $\beta$-amyloid clearance, and maintenance of the blood-brain barrier integrity (Vance et al., 2000; Mauch et al., 2001; Jiang et al., 2008; Bell et al., 2012). In humans, polymorphisms in ApoE result in the following three isoforms of the protein-ApoE2, ApoE3, and ApoE4. ApoE3 has been proposed as the "wild-type"(WT) allele because it is the most prevalent isoform in the population and is not associated with a disease phenotype (Hauser et al., 2011). The presence of ApoE4 in humans is the strongest known genetic risk factor associated with the development of late-onset Alzheimer's disease as well as poorer outcomes after traumatic brain injury, with links to dementia and cognitive function deficits (Jordan et al., 1997; Teasdale et al., 1997; Merritt and Arnett, 2016). ApoE4 transgenic mice also

DOl:http://dx.doi.org/10.1523/ENEURO.0155-18.2018

Copyright (C) 2018 Tensaouti et al.

This is an open-access article distributed under the terms of the Creative Commons Attribution 4.0 International license, which permits unrestricted use, distribution and reproduction in any medium provided that the original work is properly attributed. exhibit impaired working memory in the radial arm maze (Hartman et al., 2001), and have been shown to exhibit abnormal neuronal maturation without a shift toward astrogenesis in the dentate gyrus (Li et al., 2009).

Given that several hippocampus-related neurologic diseases are influenced by both neurogenesis and ApoE genotype and that ApoE influences developmental and adult neurogenesis, we investigated the effect of $A p o E$ human polymorphisms and ApoE deficiency on the activation of type 1 cells and their subsequent development into adult-born hippocampal neurons. To understand the myriad roles of ApoE in the brain, ApoE-deficient, human ApoE2, ApoE3, and ApoE4 targeted replacement mice have been widely used for a variety of investigations. Although it is known that ApoE influences hippocampal neurogenesis by acting as a negative regulator of proliferation in NSPCs, it remains unknown how it might affect ongoing development of adult-born neurons. Given that ApoE is expressed predominantly by astrocytes, we hypothesized that the adult newborn neuron development of dendrites and spines might be regulated by $A p o E$, where its expression in astrocytes is most abundant. Here, we demonstrate that not only does ApoE alter the proliferation of NSPCs, but it also directs changes in the complexity of mature newborn neurons at the dendritic and spine level that appear to be mediated by the close approximation of astrocytes and developing dendrites.

\section{Materials and Methods}

\section{Animals}

Experimental animals were humanely housed and cared for under the supervision of the Institute of Comparative Medicine. C57BL/6J mice (used as the wild type for all experiments) as well as ApoE-deficient mice were purchased from The Jackson Laboratory. Purchased ApoE-deficient mice were crossed with and maintained under C57BL/6J genetic background as described previously (Yang et al., 2011). ApoE3 and ApoE4 targeted replacement mice were obtained from Taconic (Table 1). All experimental procedures were in accordance with the National Institutes of Health guidelines and were approved by the Institutional Animal Care and Use Commit- 
Table 2. List of antibodies used and concentrations

\begin{tabular}{|c|c|c|c|c|}
\hline Target & Description & Provider & Dilution & RRID \\
\hline GFP & Rabbit polyclonal & Invitrogen & $1: 500$ & Catalog \#A-11122; RRID:AB_221569 \\
\hline BrdU & Rat monoclonal & Abcam & $1: 500$ & Catalog \#ab6326; RRID:AB_305426 \\
\hline GFAP & Guinea Pig polyclonal & MyBioSource & $1: 500$ & Catalog \#MBS834682 \\
\hline Ki67 & Rabbit monoclonal & Thermo Fisher Scientific & $1: 500$ & Catalog \#RM-9106-S0; RRID:AB_2341197 \\
\hline Prox1 & Rabbit polyclonal & Abcam & $1: 500$ & Catalog \#ab101851; RRID:AB_10712211 \\
\hline ApoE & Goat polyclonal & EMD Millipore & $1: 5000$ & Catalog \#AB947; RRID:AB_2258475 \\
\hline Rabbit & Biotin Goat polyclonal & Jackson ImmunoResearch Laboratories & $1: 200$ & Catalog \#111-065-003; RRID:AB_2337959 \\
\hline Goat & Biotin Donkey polyclonal & Jackson ImmunoResearch Laboratories & $1: 200$ & Catalog \#705-065-147; RRID:AB_2340397 \\
\hline Rat & A488 Donkey polyclonal & Jackson ImmunoResearch Laboratories & $1: 200$ & Catalog \#712-546-153; RRID:AB_2340686 \\
\hline Rabbit & A488 Donkey polyclonal & Jackson ImmunoResearch Laboratories & $1: 200$ & Catalog \#711-545-152; RRID:AB_2313584 \\
\hline Guinea pig & A647 Donkey polyclonal & Jackson ImmunoResearch Laboratories & $1: 200$ & Catalog \#706-605-148; RRID:AB_2340476 \\
\hline Rabbit & A647 Donkey polyclonal & Jackson ImmunoResearch Laboratories & $1: 200$ & Catalog \#711-605-152; RRID:AB_2492288 \\
\hline Biotin & A488 Streptavidin polyclonal & Jackson ImmunoResearch Laboratories & $1: 200$ & Catalog \#016-540-084; RRID:AB_2337249 \\
\hline Goat serum & Normal Goat Serum antibody & Jackson ImmunoResearch Laboratories & $5 \%$ & Catalog \#005-000-121; RRID:AB_2336990 \\
\hline Donkey serum & Normal Donkey Serum antibody & Jackson ImmunoResearch Laboratories & $5 \%$ & Catalog \#017-000-121; RRID:AB_2337258 \\
\hline
\end{tabular}

RRID, Research resource identifier (https://scicrunch.org/resources).

tee at Columbia University College of Physicians and Surgeons.

\section{Retroviral injections}

To highlight the potential physical interaction between ApoE-expressing astrocytes and adult-born granule cells in the dentate gyrus, one 6-week-old tdTomato reporter mouse (Table 1) was injected with an eGFPcre-expressing retrovirus $\left[1 \times 10^{9}\right.$ transducing units $\left.(\mathrm{TU}) / \mathrm{ml}\right]$ into the dentate gyrus [Moloney Murine Leukemia Viral vectors were generated by the GT3 Core Facility of the Salk Institute (La Jolla, CA) with funding by National Institutes of Health (NIH)-National Cancer Institute (NCl) Cancer Center Support Grant (CCSG) P30-014195, a National Institute of Neurological Disorders and Stroke (NINDS) R24 Core Grant; and funding from National Eye Institute (NEI)] inducing robust expression of tdTomato fluorescence in infected cells 4 weeks postinjection. Twenty-one 6-week-old mixed-sex mice were stereotactically injected with an enhanced green fluorescent protein (eGFP)expressing retrovirus to infect adult neural progenitors in the dentate gyrus (Moloney Murine Leukemia Viral vectors were generated by the GT3 Core Facility of the Salk Institute with funding from NIH-NCI CCSG P30-014195, an NINDS R24 Core Grant and funding from NEI). The constitutive expression of eGFP in the infected cells allowed for the tracing of infected NSPCs. One microliter of packaged GFP-expressing retrovirus $\left(1 \times 10^{9} \mathrm{TU} / \mathrm{ml}\right)$ was infused, using a microinfusion pump (KD Scientific) linked to a $10 \mu \mathrm{l}$ syringe (model \#801, Hamilton), at the rate of $0.1 \mu \mathrm{l} / \mathrm{min}$ into the dentate gyrus with the following coordinates: antero/posterior, $-2.0 /-2.5 \mathrm{~mm}$; medio/lateral, $\pm 1.55 / \pm 2 \mathrm{~mm}$; dorso/ventral, $-2.0 /-2.25 \mathrm{~mm}$.

\section{Tissue processing and immunohistochemistry}

Four weeks after retroviral injections, the time it takes for a newborn granular neuron to be mature and become integrated into the pre-existing hippocampal circuitry (Toni and Schinder, 2015), animals were deeply anesthetized with isoflurane through the whole period of perfusion. Transcardiac perfusion was performed with $50 \mathrm{ml}$ of $1 \times$ PBS, followed by $100 \mathrm{ml}$ of $4 \%$ paraformaldehyde (PFA) in $1 \times$ PBS. Whole brains were then dissected and immersed in $4 \%$ PFA $1 \times$ PBS overnight for postfixation.
Then, brains were embedded in $3 \%$ agarose $/ 1 \times$ PBS, and serial $50 \mu \mathrm{m}$ sections were cut with a vibratome (model VT1000S, Leica). All sections encompassing the hippocampus were collected sequentially in six-well plates. Free-floating sections were used for immunohistochemistry. All brain sections containing the hippocampus also had GFP-expressing adult-born granules cells, showing the efficacy of injections. All samples were kept at $-20^{\circ} \mathrm{C}$ in antifreeze solution (30\% glycerol $/ 30 \%$ ethylene glycol in PBS).

A set of sections containing every sixth slice was washed with PBS $1 \times$ to remove antifreeze solution $(3 \times 5 \mathrm{~min})$ then samples were permeabilized with $0.3 \%$ Triton $X-100 / 1 \times$ PBS (PBST; $3 \times 10 \mathrm{~min}$ ) at room temperature (RT). Samples were then blocked for $1 \mathrm{~h}$ at RT with PBST containing $5 \%$ normal goat serum (NGS) or normal donkey serum (NDS; Jackson ImmunoResearch Laboratories). Samples were then incubated with primary antibodies (Table 2, references and concentrations used) in PBST with 5\% NGS or NDS at $4^{\circ} \mathrm{C}$ overnight or at RT [with $0.02 \%(\mathrm{w} / \mathrm{v})$ sodium azide] overnight. The following day, sections were washed with PBST three times and incubated with secondary antibodies (Alexa Fluor 488, Alexa Fluor 594, Alexa Fluor 647, or biotinconjugated antibodies, Jackson ImmunoResearch Laboratories) for $3 \mathrm{~h}$ at RT. Sections were then washed three times with PBS and incubated with Alexa Fluor 488-, Alexa Fluor 594-, or Alexa Fluor 647-conjugated streptavidin antibodies (Jackson ImmunoResearch Laboratories) for $2 \mathrm{~h}$ at RT if biotin-conjugated antibodies were used. Sections were finally rinsed three times with PBS, then mounted on slides with Vectashield Mounting Medium with DAPI (catalog \#H1500 , Vector Laboratories) and sealed with coverslips. For BrdU staining, sections were denatured with $1 \mathrm{~N} \mathrm{HCl}$ for 45 min in a $37^{\circ} \mathrm{C}$ water bath before normal staining procedure.

\section{BrdU and Prox1 quantification}

BrdU was administered via intraperitoneal injection (100 $\mathrm{mg} / \mathrm{kg}$ ) for 3 consecutive days to 196 -week old wild-type C56JBL/6, ApoE-deficient, ApoE3 and ApoE4 mice from males and females, and brains were harvested for further analyses at 10 weeks of age after transcardiac perfusion as described above. To determine the number of BrdUpositive and Brdu-Prox1 double-positive cells in both the subgranular zone (SGZ) and granular cell layer (GCL) of 
the dentate gyrus, an unbiased stereological approach was used. Samples were analyzed using a Zeiss microscope (Axio Imager M2, Zeiss) with a Hamamatsu camera (Orca-R2, Hamamatsu). The cells were counted using an optical fractionator and stereological image analysis software (RRID:SCR_002526). SGZ and GCL of the dentate gyrus were traced under a $10 \times$ objective lens. The stereological software randomly selected sample grids (100 $\times 100 \mu \mathrm{m})$, and cells within the counting frames $(50 \times 50$ $\mu \mathrm{m})$ were counted under a $40 \times$ objective. To avoid artifacts that resulted from sectioning, a dissector height of $30 \mu \mathrm{m}$ was used. In addition to counting BrdU-positive cells, colocalization of BrdU and Prox1 was also quantified. The software estimated cell number by using weighted section thickness to yield an absolute number of cells in the hippocampus. Following each count, the software also calculated a coefficient of error. To ensure that the estimated counts were accurate, the coefficient of error for each included count was $<0.1$.

\section{Nestin, Ki-67, and GFAP quantification}

To determine the number of nestin-positive, Ki67positive, nestin-GFAP double-positive, and nestin-GFAPKi67 triple-positive cells in the SGZ of the dentate gyrus, 10-week-old wild-type C56JBL/6, ApoE-deficient, ApoE3, and ApoE4 mice samples (4 mice/group) were sectioned, stained, and analyzed using stereological quantification as described. The SGZ of the dentate gyrus was traced under a $20 \times$ objective lens. The stereological software randomly selected sample grids $(200 \times 200 \mu \mathrm{m})$, and cells within the counting frames $(100 \times 100 \mu \mathrm{m})$ were counted under a $63 \times$ oil-objective. To avoid artifacts that resulted from sectioning, a dissector height of $30 \mu \mathrm{m}$ was used. The software estimated cell numbers by using weighted section thickness to yield an absolute number of cells in the hippocampus. To ensure the estimated counts were accurate, the coefficient of error for each included count was $<0.1$. Representative images were acquired using a Laser Scan Confocal Microscope (TCS SP8, Leica), and images were deconvolved using Autoquant (RRID: SCR_002465).

\section{Analysis of dendritic morphology and spine density}

Immunostained sections were visualized using a Zeiss microscope (Axio Imager M2, Zeiss) with a Hamamatsu camera (Orca-R2, Hamamatsu). Stack images (1 $\mu \mathrm{m}$ interval on $z$-axis) were acquired using an optical fractionator and stereological image analysis software (RRID: SCR_002526) under a $20 \times$ objective. A minimum of 10 neurons/mouse underwent three-dimensional (3D) reconstruction using the Neurolucida360 software (RRID: SCR_001775; Dickstein et al., 2016) to analyze the morphology of those cells: measurement of the length of the proximal branch before the first division (first branch), the cumulative length of the dendritic tree, the number of nodes, and the span of the dendritic tree, and a Sholl analysis were performed.

Sholl analysis (see Figs. 3G, 5G) revealed that the number of dendrites of adult-born granule cells peaks at $\sim 100$ $\mu \mathrm{m}$ from the soma, and we found statistical differences among the different genotypes. For further investigation, spine analysis was performed on dendrites in the molecular layer $(\mathrm{ML})$ only. Image stacks were obtained using a Laser Scan Confocal Microscope (TCS SP8, Leica), under a $63 \times$ oil-objective lens, with $5 \times$ digital zoom, intervals of $0.1 \mu \mathrm{m}$ along the $z$-axis, leading to a pixel size of $57.21 \mathrm{~nm}$ (numerical aperture $=1.44$; resolution $=512 \times 512$; frame average $=4$ ). Images were deconvolved using Autoquant (RRID:SCR_002465), $10 \mu \mathrm{m}$ dendritic fragments were randomly picked for tracing (one fragment/ dendrite) and were analyzed by an experimenter blindly using the automated software Neurolucida 360 (RRID: SCR_001775), which performed 3D analysis of dendritic length and spine number (Dickstein et al., 2016). After automated detection of spines, a manual examination was performed (i.e., adding, deleting, merging, or splitting identified objects). Because of spherical aberrations, objects on the $z$-axis appeared blurred and smeared, potentially leading to an underestimation of the spine density, added to the fact that the diameters of dendrites vary too, we elected to express the number of spines as a function of the fragment length instead of using its surface, which could potentially lead to confounding results (Dumitriu et al., 2011).

\section{Statistical analysis}

All statistical analyses were performed using GraphPad Prism (RRID:SCR_015807). The normality of data were assessed using the Shapiro-Wilk test, and variance was assessed using Levene's test. Results are presented as the mean \pm SEM. Statistical details are presented in text and summarized in Table 3. The unpaired Student's $t$ test was used to test for differences between wild-type and ApoE-deficient animals, and between ApoE3 and ApoE4 animals since these two groups [ApoE knock-out (KO)/ wild-type and ApoE3/ApoE4 were on distinct genetic backgrounds]. Two-way ANOVA with Fisher's least significant difference (LSD) post hoc test was used to test differences in Sholl analysis. The effect size was calculated using Cohen's $d$ statistic. A value of $p<0.05$ was considered statistically significant.

\section{Results}

\section{ApoE expression during dentate gyrus neuronal maturation}

ApoE demonstrates widespread expression in the dentate gyrus, both in nestin-expressing neural stem and progenitor cells in the subgranular zone as well as GFAPexpressing astrocytes in the hilus and molecular layer (Fig. $1 A-H)$. To determine the expression of ApoE in approximation to the dendritic arborizations of adult-born neurons, a retrovirus expressing Cre was injected stereotactically into the dentate gyrus subgranular zone of ROSA-26 tdTomato reporter mice to infect dividing NSPCs, which then express tdTomato. Four weeks after injection, mice were sectioned and stained for ApoE and GFAP to demonstrate astrocytic expression of ApoE. We observed that ApoE-expressing astrocytes are in very close proximity and appear to be physically interacting with the dendrites of just maturing adult-born neurons (Fig. 1/-P). 
Table 3. Summary of statistics.

\begin{tabular}{|c|c|c|c|c|c|c|c|c|}
\hline & Table analyzed & Statistical test & $p$ value & Size effect & $t$ value & df & $R^{2}$ & $F$ value \\
\hline \multirow[t]{12}{*}{ WT vs KO } & BrdU counts & Unpaired Student's $t$ test & 0.8469 & 0.60 & 0.494 & 8 & 0.029 & $\begin{array}{l}9.72 \\
p=0.049\end{array}$ \\
\hline & BrdU-Prox1 colocalization & Unpaired Student's $t$ test & 0.6784 & 0.23 & 0.3652 & 8 & 0.016 & $\begin{array}{l}5.473 \\
p=0.1284\end{array}$ \\
\hline & $\%$ Newborn neurons & Unpaired Student's $t$ test & 0.2214 & 0.19 & 0.2983 & 8 & 0.011 & $\begin{array}{l}1.266 \\
p=0.8249\end{array}$ \\
\hline & Nestin-Ki67-GFAP & Unpaired Student's $t$ test & 0.0376 & 1.88 & 2.658 & 6 & 0.5408 & $\begin{array}{l}8.054 \\
p=0.1204\end{array}$ \\
\hline & Ki67 & Unpaired Student's $t$ test & 0.0333 & 2.47 & 2.75 & 6 & 0.5576 & $\begin{array}{l}1.015 \\
p=0.9903\end{array}$ \\
\hline & Length of the first branch & Unpaired Student's $t$ test & 0.1658 & 0.23 & 1.393 & 149 & 0.0128 & $\begin{array}{l}1.284 \\
p=0.2817\end{array}$ \\
\hline & Number of nodes & Unpaired Student's $t$ test & 0.0001 & 0.65 & 3.939 & 149 & 0.0943 & $\begin{array}{l}1.617 \\
p=0.0406\end{array}$ \\
\hline & Sholl analysis & & & & & & & \\
\hline & Intersection effect & Two-way ANOVA & $<0.0001$ & & & & & $F_{(26,4023)}=2.488$ \\
\hline & Row effect & Two-way ANOVA & $<0.0001$ & & & & & $F_{(26,4023)}=163.7$ \\
\hline & Column effect & Two-way ANOVA & $<0.0001$ & & & & & $F_{(1,4023)}=37.46$ \\
\hline & Spine density & Unpaired Student's $t$ test & $<0.0001$ & 1.13 & 4.163 & 107 & 0.1394 & $\begin{array}{l}1.016 \\
p=0.9479\end{array}$ \\
\hline \multirow[t]{10}{*}{ E3 vs E4 } & BrdU Counts & Unpaired Student's $t$ test & 0.8961 & 0.094 & 0.135 & 7 & 0.002612 & $\begin{array}{l}2.668 \\
p=0.4463\end{array}$ \\
\hline & BrdU-Prox1 colocalization & Unpaired Student's $t$ test & 0.5838 & 0.40 & 0.574 & 7 & 0.04498 & $\begin{array}{l}3.759 \\
p=0.3053\end{array}$ \\
\hline & $\%$ Newborn neurons & Unpaired Student's $t$ test & 0.1097 & 1.22 & 1.832 & 7 & 0.324 & $\begin{array}{l}1.234 \\
p=0.8143\end{array}$ \\
\hline & Number of nodes & Unpaired Student's $t$ test & 0.0007 & 0.59 & 3.472 & 142 & 0.07826 & $1.408, p=0.1542$ \\
\hline & DA cumulative length & Unpaired Student's $t$ test & $<0.0001$ & 0.7 & 4.127 & 142 & 0.1071 & $1.783, p=0.0166$ \\
\hline & $\begin{array}{l}\text { DA Span } \\
\text { Sholl analysis }\end{array}$ & Unpaired Student's $t$ test & 0.5978 & 0.09 & 0.528 & 142 & 0.001965 & $1.296, p=0.2749$ \\
\hline & Intersection effect & Two-way ANOVA & 0.0013 & & & & & $F_{(26,3834)}=2.048$ \\
\hline & Row effect & Two-way ANOVA & $<0.0001$ & & & & & $F_{(26,3834)}=144.7$ \\
\hline & Column effect & Two-way ANOVA & $<0.0001$ & & & & & $F_{(1,3834)}=112.8$ \\
\hline & Spine density & Unpaired Student's $t$ test & $<0.0001$ & 1.13 & 5.278 & 88 & 0.2405 & $\begin{array}{l}2.08 \\
p=0.0173\end{array}$ \\
\hline
\end{tabular}

D, Dendritic arborization. Cohen's $d$ value was calculated as $d=$ Mean $1-$ Mean2 $/$ SDpooled with SDpooled $=\sqrt{S D_{1}^{2}+S D_{2}^{2} / 2}, d=0.2, d=0.5$, and $d=$ 0.8 corresponding to small, medium, and large size effects, respectively

\section{Type 1 neural stem and progenitor proliferation in wild-type and ApoE-deficient mice}

To investigate the effect of ApoE on neuronal survival and differentiation in the hippocampus, BrdU (100 mg/kg) was administered via intraperitoneal injection for three consecutive days to 6-week-old wild-type and ApoEdeficient mice, and brains were harvested for further analysis 4 weeks later, the time it takes for immature neurons to express mature neuronal markers. Using unbiased stereology, BrdU-positive cells, and BrdU-Prox1 doublepositive cells, representing dividing cells that became granular neurons, were quantified; and total counts were estimated. Additionally, to determine what percentage of newborn hippocampal cells became mature granular neurons, the number of BrdU-Prox1 colocalized cells was divided by the total number of BrdU-positive cells and multiplied by 100 . No significant differences were found between wild-type and ApoE-deficient mice for total BrdU-positive cells (Fig. 2A; WT, $5368 \pm 474.2$; KO, 5614 \pm 152.1; unpaired Student's $t$ test), BrdU-Prox1 colocalization (Fig. 2B; WT, $4320 \pm 415.5$; KO, $4485 \pm 177.6$; unpaired Student's $t$ test), or for the percentage of new- 


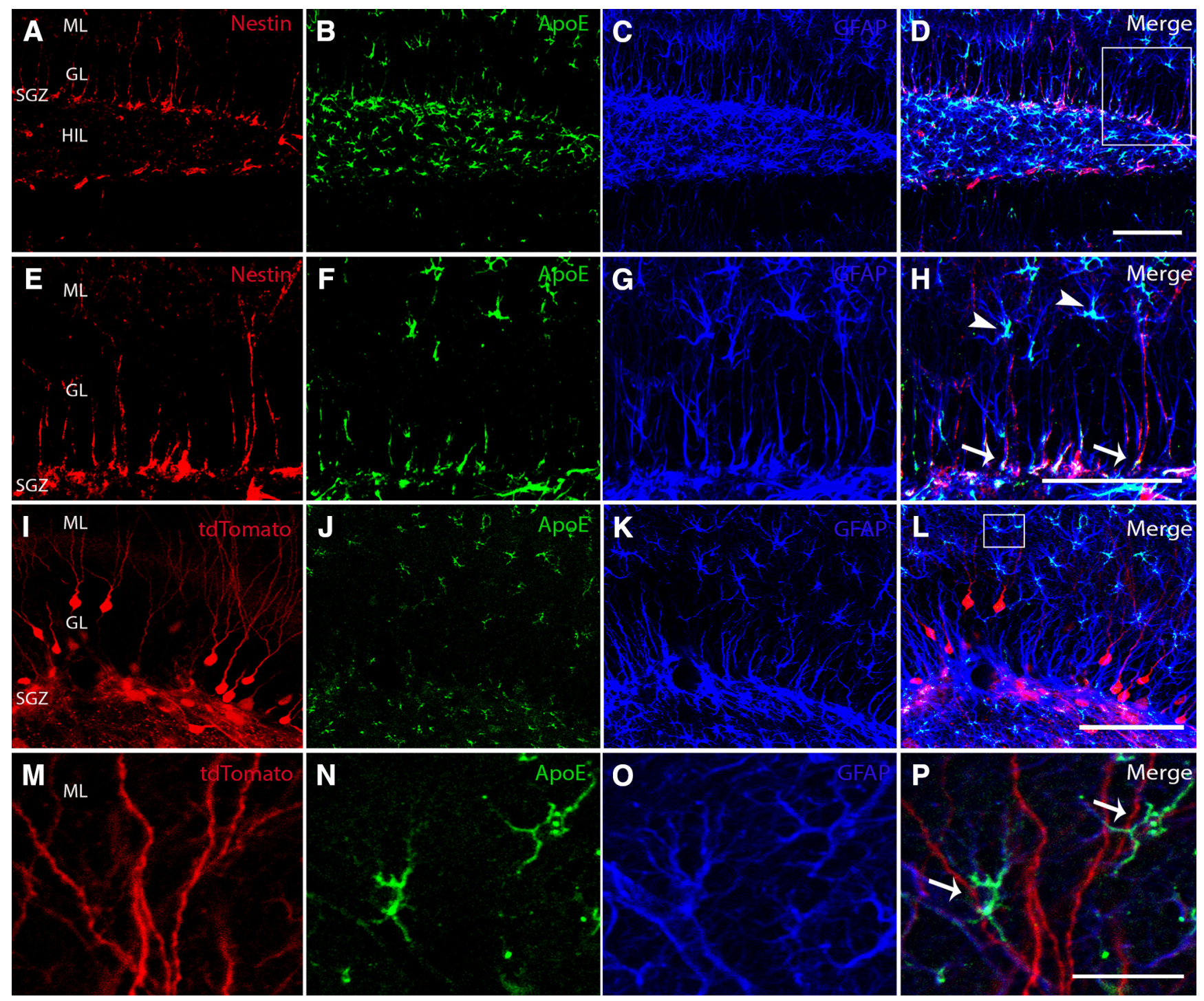

Figure 1. Type 1 NSPCs express nestin, ApoE, and GFAP, while mature granule cells make connections with astrocytes expressing ApoE and GFAP. A-D, Representative confocal images of the dentate gyrus illustrate nestin-expressing (red), ApoE-expressing (green), and GFAP-expressing (blue) cells, along with a merged image of all channels. $\boldsymbol{E}-\boldsymbol{H}$, High-power representative confocal images taken from the region in the inset in $\boldsymbol{D}$ illustrate nestin-expressing (red), ApoE-expressing (green), and GFAP-expressing (blue) cells, along with a merged image of all channels. White arrows indicate nestin-ApoE-GFAP triple-positive cells, representing type 1 NSPCs. I-L, Representative confocal images of the dentate gyrus illustrate tdTomato-expressing (red), ApoE-expressing (green), and GFAP-expressing (blue) cells, along with a merged image of all channels. $\boldsymbol{M}-\boldsymbol{P}$, High-power representative confocal images taken from the region in the inset in $\boldsymbol{L}$ illustrate tdTomato-expressing (red), ApoE-expressing (green), and GFAP-expressing (blue) cells, along with a merged image of all channels. White arrows indicate GFAP and ApoE coexpressing astrocytes. GL, Granule layer; HIL, hilus. Scale bars: $\boldsymbol{D}, 100 \mu \mathrm{m} ; \boldsymbol{H}, 75 \mu \mathrm{m} ; \boldsymbol{L}, 100 \mu \mathrm{m} ; \boldsymbol{P}, 25 \mu \mathrm{m}$.

born neurons (Fig. 2C; WT, $80.30 \pm 1.136$; KO, $79.79 \pm$ 1.278; unpaired Student's $t$ test), indicating that ApoE deficiency does not impair the ability of newborn hippocampal cells to survive and differentiate into granular neurons 4 weeks after BrdU administration.

Because there was no significant change in the ability of newborn hippocampal cells to survive and differentiate into neurons in the absence of $\mathrm{ApoE}$, and because previous studies have determined that ApoE expression is high in type 1 NSPCs (Hong et al., 2016), the proliferation of NSPCs was next analyzed to determine whether ApoE deficiency affects type 1 NSPC activation specifically.
Four samples from each genotype were sectioned and immunostained for nestin, Ki67, and GFAP, and were quantified using unbiased stereology. Representative images of the SGZ are shown for wild-type and ApoEdeficient mice (Fig. 2D-K). Compared with wild-type controls, ApoE-deficient brains exhibited significantly reduced Ki67 staining (Fig. 2L; WT, $4477 \pm 524.0 ; \mathrm{KO}, 2433$ \pm 528.0; unpaired Student's $t$ test: $p=0.033, d=2.47$, $t=2.75$ ), indicating a reduction in overall proliferation at 10 weeks of age. Additionally, in the absence of ApoE, there were significantly fewer nestin-GFAP colocalized cells in the dentate gyrus (Fig. 2M; WT, $15430 \pm 1088.0$; 
A

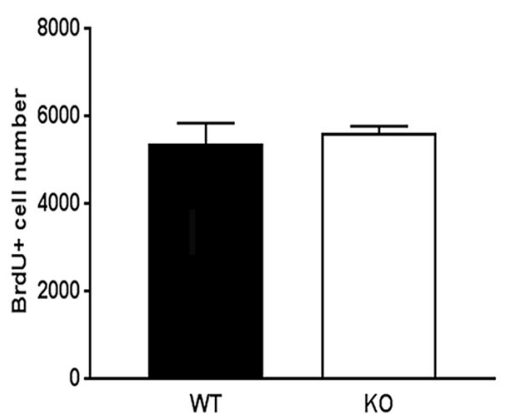

B

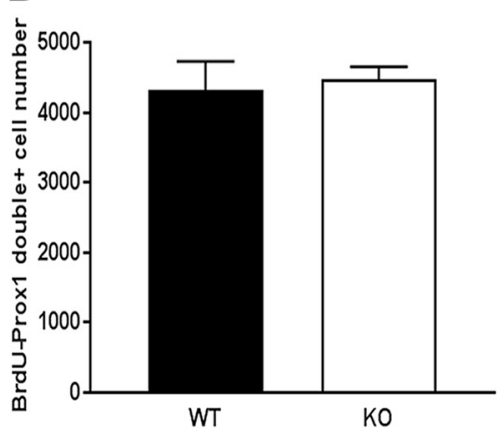

C

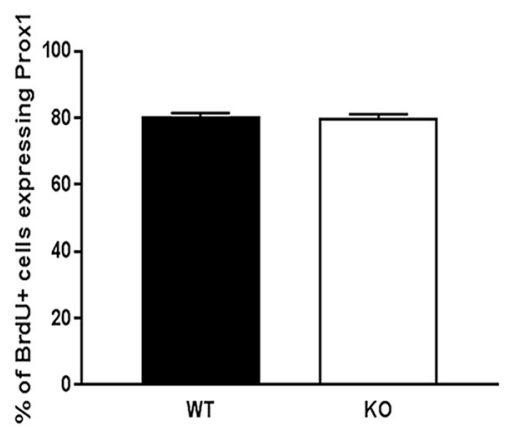

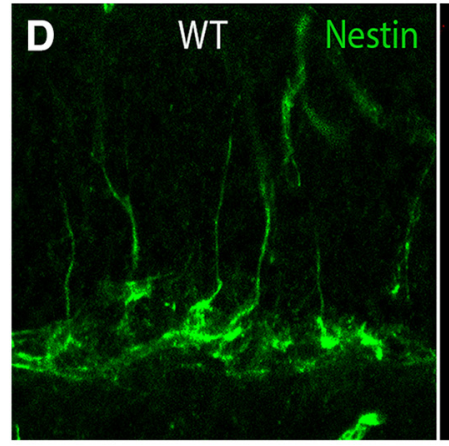
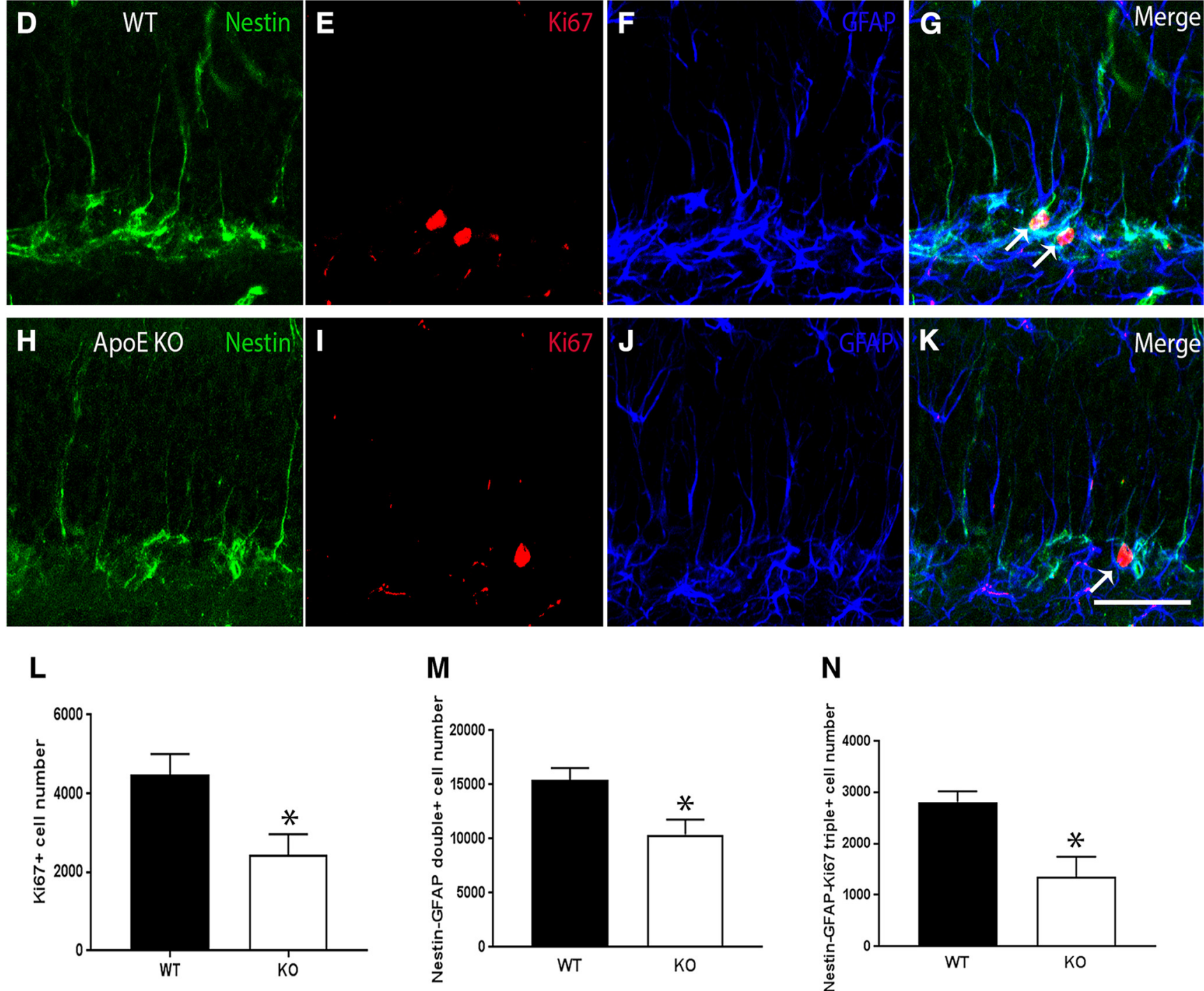

Figure 2. ApoE deficiency leads to a decrease in overall proliferation, the number of type 1 NSPCs, and type 1 NSPC proliferation in the dentate gyrus, while leaving the ability of newborn cells to survive and differentiate into granular neurons unimpaired. $\boldsymbol{A}-\boldsymbol{C}$, Unbiased stereological quantification of the number of BrdU-positive cells $(\boldsymbol{A})$, the number of BrdU-Prox1 double-positive cells $(\boldsymbol{B})$, and the percentage of BrdU-positive cells that also express Prox1 (C). D-K, Representative confocal images of the SGZ in WT and ApoE-deficient mice illustrate nestin-expressing (green), Ki67-expressing (red), and GFAP-expressing (blue) cells, along with a merged image of all channels. White arrows indicate nestin-Ki67-GFAP triple-positive cells, representing actively proliferating type 1 NSPCs. $\boldsymbol{L}-\boldsymbol{N}$, Unbiased stereological quantification of the number of Ki67-positive cells ( $\boldsymbol{L}$ ), the number of nestin-GFAP doublepositive cells $(\boldsymbol{M})$, and the number of nestin-GFAP-Ki67 triple-positive cells $(\boldsymbol{N})$. Results are expressed as the mean \pm SEM. Unpaired Student's $t$ tests, $* p<0.05$. Scale bar, $\boldsymbol{K}, 50 \mu \mathrm{m}$. 
KO, $10390 \pm 1393.0$; unpaired Student's $t$ test: $p=$ $0.0291, d=2.02, t=2.85$ ), representing a reduction in the total number of NSPCs. Further, ApoE-deficient samples had significantly fewer nestin-GFAP-Ki67 triple-positive cells (Fig. 2N; WT, $3592 \pm 295.0 ; \mathrm{KO}, 1351 \pm 280$; unpaired Student's $t$ test: $p=0.0376, d=1.88, t=2.66$ ), a decrease suggesting that ApoE deficiency specifically affects NSPC proliferation.

\section{ApoE deficiency impairs the dendritic complexity of adult-born dentate gyrus neurons}

After proliferating in the SGZ of the dentate gyrus, NSPCs migrate short distances to the inner segment of the GCL and start to expand and develop their dendritic trees into the ML (Kempermann et al., 2015). To assess the effect of ApoE on the maturation of adult-born granule cell dendritic trees in the dentate gyrus, 6-week-old C56JBL/6 mice ( $n=4$ mice, 78 neurons) and ApoEdeficient mice ( $n=7$ mice, 73 neurons) were stereotactically injected with a GFP-expressing retrovirus and killed 4 weeks after injection to make 3D reconstructions. The morphology of these neurons was analyzed based on the length of the proximal branch before the first division (the first branch), the cumulative length of the dendritic tree, the number of nodes (every time a branch divides), the span of the dendritic tree, and Sholl analysis.

Representative pictures of 3D-reconstructed adultborn granule cells are shown for both WT and ApoEdeficient mice (Fig. $3 A, B$ ). We did not observe any difference in the location of newborn granule cells in the dentate gyrus of WT and ApoE-deficient mice, because, apart from rare exceptions, all adult-born granule cells were found in the inner one-third of the GCL. No significant differences were found in the length of the first branch between WT and ApoE-deficient mice (Fig. 3C; WT, $36.41 \pm 1.97 \mu \mathrm{m} ; \mathrm{KO}, 37.59 \pm 2.19 \mu \mathrm{m}$; unpaired Student's $t$ test). After the first dendritic division, new dendrites continue to grow in the GCL and ML, sensing the environment and bifurcating several times (Kempermann et al., 2015). We found a significant decrease in the number of nodes in ApoE-deficient mice (6.25 \pm $0.24 \mu \mathrm{m})$ compared with WT mice $(7.77 \pm 0.29 \mu \mathrm{m}$; Fig. $3 D$; unpaired Student's $t$ test: $p=0.001, d=0.65, t=$ 3.94). However, no significant differences have been found in the total dendritic length (Fig. 2E; WT, $875.2 \pm$ $26.01 \mu \mathrm{m}$; KO, $785.5 \pm 25.76 \mu \mathrm{m}$; unpaired Student's $t$ test). We also found that the dendritic tree span (42.54 $\pm 1.45^{\circ}$ ) of ApoE-deficient mice was significantly reduced compared with that of WT mice $\left(51.47 \pm 2.58^{\circ}\right.$; Fig. 3F; unpaired Student's $t$ test: $p=0.0035, d=0.49$, $t=2.97$.

We then analyzed the distribution of adult-born granule cell dendrites in the dentate gyrus by performing Sholl analysis (Fig. $3 A, B$, representative pictures). Two-way ANOVA revealed a highly significant effect of the genotype and the distance from the soma on the number of intersections $\left(p<0.0001, F_{(1,4023)}=37.46\right.$ and $p<$ $0.0001, F_{(26,4023)}=163.7$, respectively), as well as a highly significant interaction between those two factors $(p<$ $\left.0.0001, F_{(26,4023)}=2.488\right)$. Post hoc analysis did not demonstrate a change in the number of proximal dendrites (from 10 to $50 \mu \mathrm{m}$ ) among the different genotypes (Fig. 3G; uncorrected Fisher's LSD post hoc tests) consistent with the fact that we did not find any difference in the occurrence of the first dendritic division (Fig $3 C$ ). However, we found that ApoE-deficient adult-born neurons had significantly fewer dendritic branches from 50 to $100 \mu \mathrm{m}$ from the soma when compared to WT mice. For both groups, the dendrites reached the $\mathrm{ML}$ and the number of dendrites peaked at $100 \mu \mathrm{m}$. Furthermore, ApoEdeficient granule cells demonstrated significantly fewer interactions on Sholl analysis than WT cell at 100 to 150 $\mu \mathrm{m}$ from the soma (Fig. 3G; uncorrected Fisher's LSD post hoc tests). No differences were observed $150 \mu \mathrm{m}$ away from the soma.

\section{Hippocampal neuronal maturation in ApoE3 and ApoE4 mice}

While there were no significant differences between ApoE-deficient and wild-type samples in the ability of newborn hippocampal cells to survive and differentiate into granular neurons, we next used unbiased stereological quantification to determine whether the presence of either the human ApoE3 or ApoE4 isoform affects these processes ( $n=5$ mice for ApoE3; $n=4$ mice for ApoE4). Similar to the wild-type and ApoE-deficient mice, no significant differences were found between ApoE3 and ApoE4 mice for total BrdU-positive cells (E3, $4032 \pm$ 368.3; E4, $3968 \pm 252.1$; unpaired Student's $t$ test, results not shown), BrdU-Prox1 colocalization (E3, $3216 \pm 290.3$; $E 4,3009 \pm 167.4$; unpaired Student's $t$ test) or for the percentage of newborn neurons (E3, $79.79 \pm 1.322$; E4, $75.98 \pm 1.642$, unpaired Student's $t$ test), suggesting that the presence of either human isoform does not differentially impair the ability of newborn hippocampal cells to survive and differentiate into granular neurons 4 weeks after BrdU administration.

As there were no significant differences in neuronal survival and maturation between ApoE3 and ApoE4 mice, levels of type 1 NSPC proliferation were next studied to determine whether the human isoforms had differing effects on type 1 NSPC activation specifically. Four samples from each genotype were sectioned and immunostained for nestin, Ki67, and GFAP, and analyzed using unbiased stereological quantification. Unlike what we observed in wild-type and ApoE-deficient mice, there was no significant difference in Ki67 counts between ApoE3 and ApoE4 mice (E3, $2969 \pm$ 224.8; E4, $3476 \pm 362.5$; unpaired Student's $t$ test), indicating that the human isoforms do not differentially affect overall proliferation at 10 weeks of age. In addition, there was no significant difference in nestin-GFAP colocalization (E3, $11740 \pm$ 1410.0; E4, $9887 \pm 1292.1$; unpaired Student's $t$ test) indicating similar numbers of type 1 NSPCs in each genotype. Finally, there was no significant difference in the number of nestin-GFAP-Ki67 triple-positive cells between genotypes (E3, $1744 \pm 187.6$; E4, $1980 \pm 256.9$; unpaired Student's $t$ test), suggesting that the human isoforms lead to similar levels of NSPC proliferation. 
A

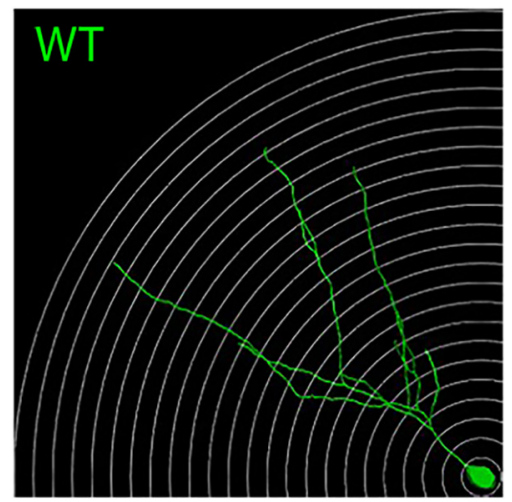

C

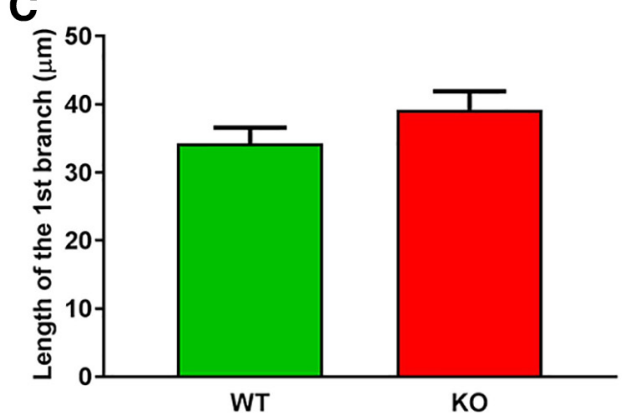

E

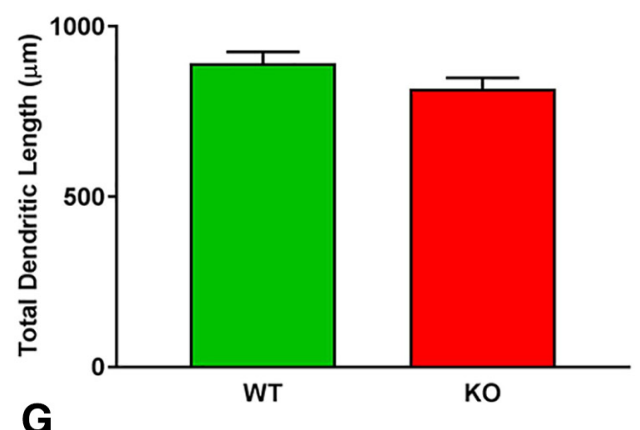

B

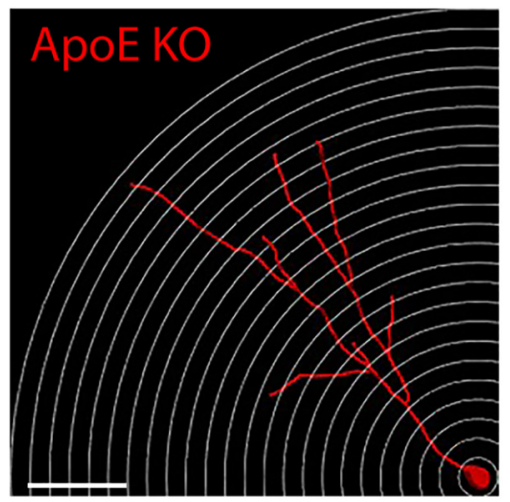

D

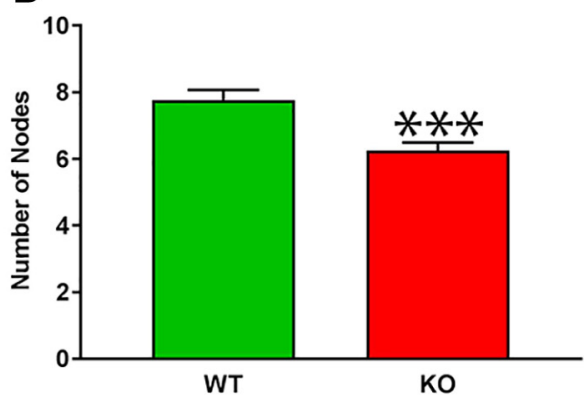

F

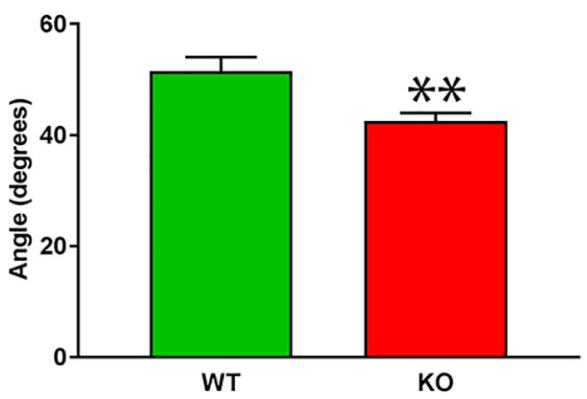

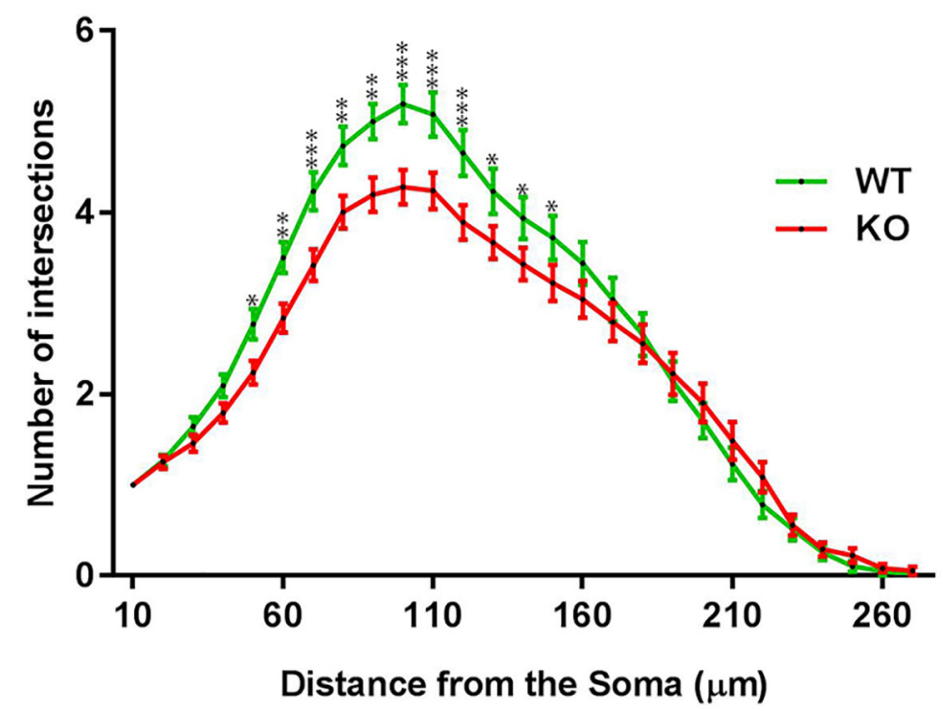

Figure 3. The complexity as well as the total dendritic length of ApoE-deficient adult-born granule cells are significantly reduced. $\boldsymbol{A}$, $\boldsymbol{B}$, Representative 3D-reconstructed adult-born granule cells (4 weeks after stereotactic injections), one circle every $10 \mu \mathrm{m}$. $\boldsymbol{C}$, Distance between the soma and the first dendritic division (in $\mu \mathrm{m}$ ). $\boldsymbol{D}$, Nodes were defined as the number of divisions of dendritic 
continued

branches. $\boldsymbol{E}$, Cumulative length of dendritic arborization (in $\mu \mathrm{m}$ ). $\boldsymbol{F}$, Span of the dendritic arborization when projected on two dimensions. G, Sholl analysis: the number of dendritic intersections as a function of the distance from the soma: wild type, 78 neurons/4 mice; ApoE-deficient, 73 neurons/7 mice. Results are expressed as the mean \pm SEM. Independent $t$ test and two-wayANOVA with uncorrected Fisher's LSD post hoc tests were used: $* p<0.05, * * p<0.01, * * * p<0.001$. Scale bar, $\boldsymbol{B}, 50 \mu \mathrm{m}$.

\section{Adult-born granule neurons in ApoE4 mice demonstrate less complexity than that seen in ApoE3}

After observing an effect of ApoE deficiency on the dendritic morphology of adult-born granule cells in the dentate gyrus (i.e., reduced dendritic complexity, span, and number of branches in both $\mathrm{GCL}$ and $\mathrm{ML}$ ), we next investigated the effect of human ApoE3 and ApoE4 on the development of mouse dentate gyrus adult born granule cell dendritic trees. Representative 3D-reconstructed adult-born granule cells are shown (Fig. 4A,B) for both ApoE3 mice ( $n=6$ mice, 75 neurons) and ApoE4 mice ( $n$ $=4,69$ neurons). No significant differences were found in the length of the first branch (Fig. 4C; E3, $38.99 \pm 2.14$ $\mu \mathrm{m}$; E4, $39.57 \pm 2.11 \mu \mathrm{m}$; unpaired Student's $t$ test). However, a significant decrease in the number of nodes was observed in ApoE4 mice (6.37 $\pm 0.19 \mu \mathrm{m})$ compared with ApoE3 mice (7.59 $\pm 0.22 \mu \mathrm{m}$; Fig. 4D; unpaired Student's $t$ test: $p=0.0007, d=0.59, t=3.47$ ). We also found that ApoE4 total dendritic length was significantly reduced $(729.6 \pm 24.75 \mu \mathrm{m})$ when compared with ApoE3 mice (921.4 $\pm 31.57 \mu \mathrm{m}$; Fig. 4E; unpaired Student's $t$ test: $p<0.0001, d=0.7, t=4.13$ ), which corresponded to an overall $20 \%$ decrease. No significant differences were observed in the span of ApoE3 and ApoE4 dendritic trees (Fig. 4F; E3, $50.69 \pm 1.69 \mu \mathrm{m}$; E4, $52.42 \pm 1.99 \mu \mathrm{m}$; unpaired Student's $t$ test).

We then analyzed the distribution of dendritic trees for adult-born granule cells in the dentate gyrus by performing Sholl analysis (Fig. 4A,B). Two-way ANOVA revealed a highly significant effect of genotype and the distance from the soma on the number of intersections $(p<0.0001$, $F_{(1,3834)}=112.8$; and $p<0.0001, F_{(26,3834)}=144.7$, respectively), as well as a significant interaction between those two factors $\left(p=0.0013, F_{(26,3834)}=2.048\right)$. Post hoc tests did not demonstrate differences in the number of proximal dendrites (from 10 to $70 \mu \mathrm{m}$ ) between the different genotypes (Fig. 4G; uncorrected Fisher's LSD post hoc tests). However, we observed that ApoE4 adultborn neurons had significantly fewer dendritic branches from 70 to $100 \mu \mathrm{m}$ from the soma when compared with ApoE3 neurons. For both groups, the dendrites reached the molecular layer, and the number of dendrites peaked at $100 \mu \mathrm{m}$ for ApoE4 and at $110 \mu \mathrm{m}$ for ApoE3. In addition, ApoE4 granule neurons demonstrated significantly fewer dendrites than ApoE3 neurons at 100-220 $\mu \mathrm{m}$ from the soma (Fig. 4G; uncorrected Fisher's LSD post hoc tests).

\section{Distribution of adult-born granule cells as a function of their complexity}

During the acquisition and reconstruction of adult-born granule cells, we observed that the level of complexity of those neurons was heterogeneous. To quantify this obser- vation, we divided the reconstructed neurons as a function of the number of nodes, and we expressed the result as a percentage of the sample (Fig. 5B). We demonstrated that 4 weeks after retroviral injections, the average number of nodes of wild-type and ApoE3 adult-born granule cell dendrites is approximately eight (respectively, $7.77 \pm 0.29$ and $7.87 \pm 0.27 \mu \mathrm{m}$; Figs. $2 C, 4 C$ ). One observed difference is that ApoE-deficient and ApoE4 mice demonstrate a higher proportion of adult-born granule cells with four nodes (18.84\% and 19.18\%, respectively; Fig. 5A,B) compared with wild-type and ApoE3 (respectively, 5.13\% and 5.33\%). Consistent with this, we observed that $\sim 50 \%$ of wild-type and ApoE3 adult-born granule cell dendritic trees had more than eight nodes compared with $36 \%$ for ApoE4 and $26 \%$ for ApoE-deficient mice. Therefore, the dendritic trees in both wild-type and ApoE3 newborn neurons were more complex than the ones in ApoE-deficient and ApoE4 adult newborn neurons.

\section{ApoE regulates spine density}

We next investigated the effect of ApoE deficiency and human ApoE isoforms on the density of spines. Because Sholl analysis revealed that the significant difference of dendritic branches was observed at $\sim 100 \mu \mathrm{m}$ from the soma, we reconstructed dendritic fragments that were 70-120 $\mu \mathrm{m}$ away from the soma, and analyzed the spine density on randomly selected $10 \mu \mathrm{m}$ fragments. Adultborn granular neurons in the dentate gyrus of ApoEdeficient mice demonstrated a significantly reduced spine density $(4.22 \pm 0.12$ spines $/ \mu \mathrm{m})$ when compared with wild-type mice (Fig. 5C,D; $4.90 \pm 0.11$ spines/ $\mu \mathrm{m}$; unpaired Student's $t$ test: $p<0.0001, d=1.13$; Fig. $5 C 1, C 2, D)$. Similarly, ApoE4 adult-born granule cells also demonstrated a significant decrease in spine density (3.57 $\pm 0.21 \mathrm{spines} / \mu \mathrm{m}$ ) when compared with ApoE3 mice (Fig. $5 E, F ; 4.96 \pm 0.15$ spines/ $\mu \mathrm{m}$; unpaired Student's $t$ test: $p$ $<0.0001, d=1.13$; Fig. 5E1,E2,F ). Together, those findings demonstrate that the dendritic development of adult-born granule cells in the ApoE-deficient and ApoE4 mouse dentate gyrus is highly impaired.

\section{Discussion}

In this study, we demonstrate that ApoE deficiency leads to a decrease in both the number and activation of type 1 NSPCs in the 10-week-old mouse dentate gyrus. Both ApoE deficiency and ApoE4 lead to less complex mature granule cells in the dentate gyrus as well as reduced spine density at 10 weeks of age. Together, these findings highlight the crucial role of ApoE in adult mouse neurogenesis. We did not perform direct comparisons between wild-type rodent ApoE with human ApoE3 (the predominant and presumed human wild-type allele) and rodent ApoE-deficient and human ApoE4 (the less common and potentially pathogenic). Instead, we aimed to 
A

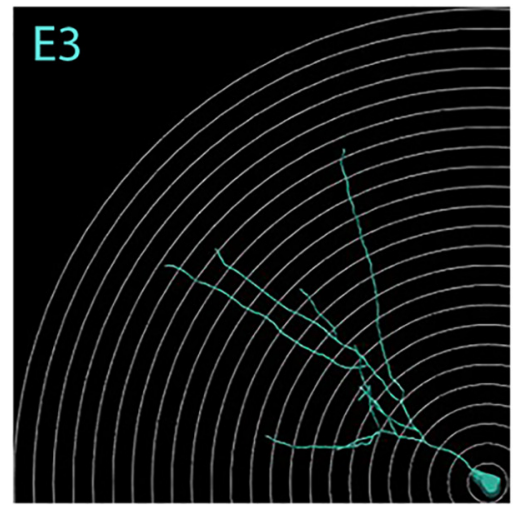

C

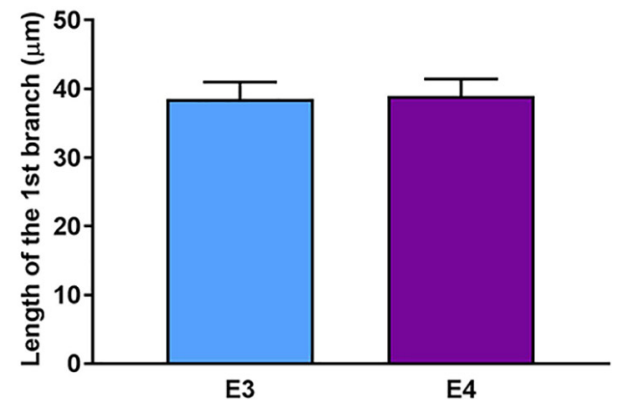

E

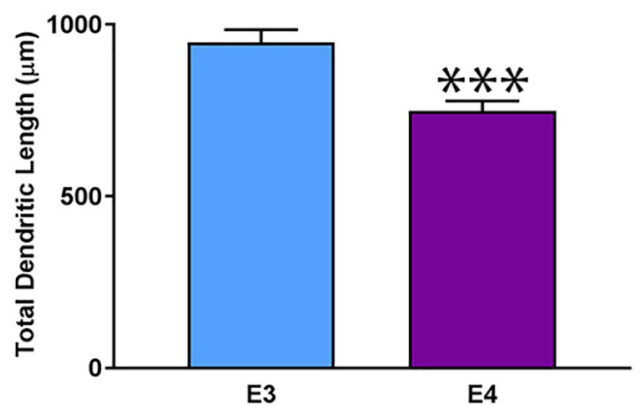

B

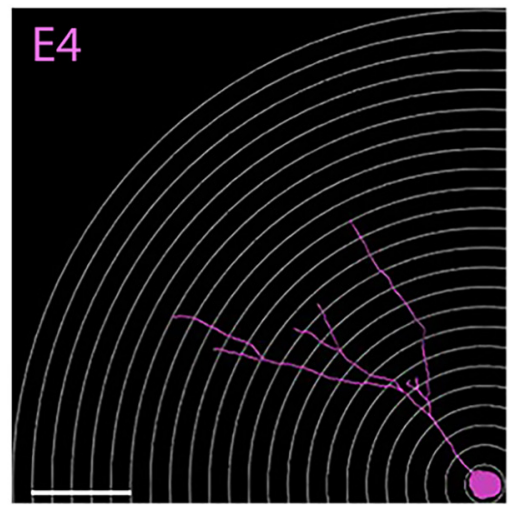

D

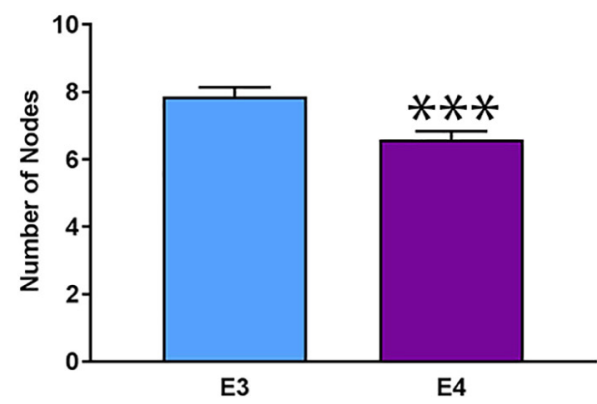

F

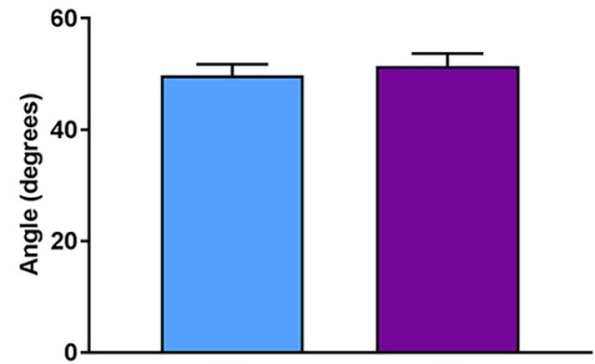

E3

E4

G

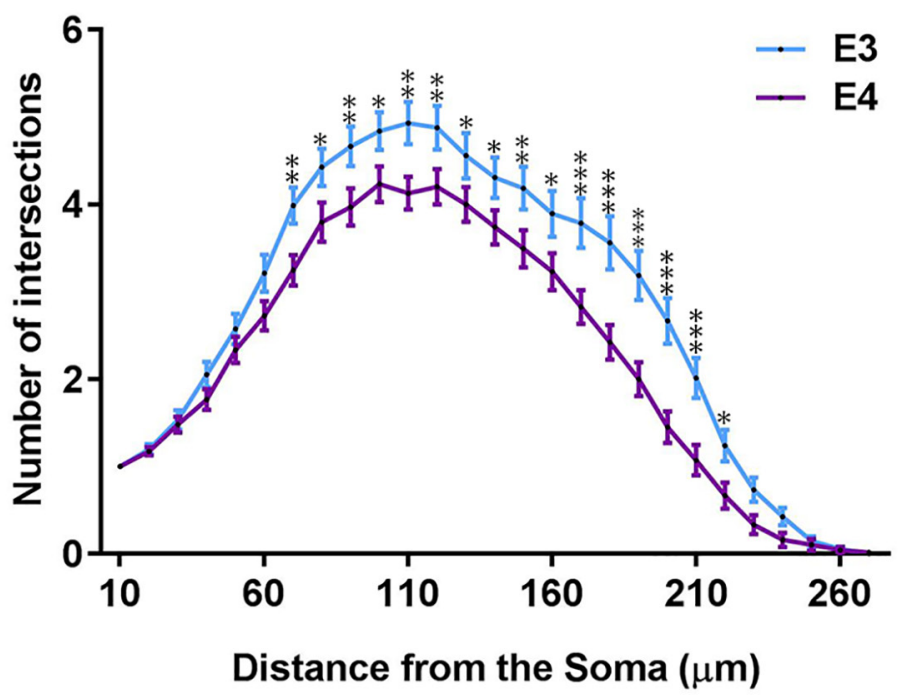

Figure 4. The complexity and the total dendritic length of ApoE4 adult-born granule cells are significantly reduced. $\boldsymbol{A}$, $\boldsymbol{B}$, Representative 3D-reconstructed adult-born granule cells (4 weeks after stereotactic injections), one circle every $10 \mu \mathrm{m}$. Representative picture of 4-week-old retrovirally labeled adult-born granule cells in the dentate gyrus. $\boldsymbol{C}$, Distance between the soma and the 
continued

first dendritic intersection (in $\mu \mathrm{m}$ ). $\boldsymbol{D}$, Nodes defined as the number of divisions of dendritic branches. $\boldsymbol{E}$, Cumulative length of dendritic arborizations (in $\mu \mathrm{m}$ ). $\boldsymbol{F}$, Span of the dendritic arborizations when projected on two dimensions. $\mathbf{G}$, Sholl analysis: number of dendritic intersections as a function of the distance from the soma. ApoE3, 75 neurons/6 mice; ApoE4, 69 neurons/4 mice. Results are expressed as the mean \pm SEM. Independent $t$ test and two-way ANOVA with uncorrected Fisher's LSD post hoc tests were used: $* p<0.05, * * p<0.01, * * * p<0.001, * * * * p<0.0001$. Scale bar, $\boldsymbol{B}, 50 \mu \mathrm{m}$.

investigate how dentate gyrus adult neurogenesis was affected in the absence of rodent ApoE and between two alleles of human ApoE. The significance of direct comparisons between species is unknown; therefore, we opted not to make them.

Adult hippocampal neurogenesis begins with the activation of quiescent type 1 NSPCs. By performing in vitro clonal analysis and in vivo unbiased stereology quantification, it has been shown that the total absence of ApoE results in an increased number of active type 1 NSPCs (Yang et al., 2011). The constitutive activation of type 1 NSPCs in ApoE-null mice depletes available type 1 NSPCs over time. Consistent with these findings, we observed a reduction in the number of both total type 1 NSPCs and active type 1 NSPCs in the absence of ApoE. Although different markers were used to determine the number of NSPCs, a similar reduction of NSPCs was observed in another study when older mice were analyzed (Li et al., 2009). Therefore, our present observations are consistent with the requirement of ApoE for the maintenance of quiescent status to prevent the depletion of available NSPCs.

Interestingly, the quantification of BrdU-positive cells and Ki67-expressing cells revealed a difference in ApoEdeficient mice with a significant decrease in Ki67expressing cells and BrdU-positive cells remaining the same compared with WT mice. It is noteworthy that injections of BrdU were given at 6 weeks of age and observed at 10 weeks of age when Ki67 was detected. A previous study (Yang et al., 2011) suggested that ApoE stimulates neural proliferation in mice that are 1 month of age, while leading to decreased proliferation in mice that are $\geq 2$ months of age, reinforcing the idea that ApoE acts as a negative regulator of neurogenesis and only leads to decreased proliferation at later ages when the progenitor pool is depleted. We hypothesize that we observed no change in BrdU incorporation in mice injected at 6 weeks of age because they were at a time when the effect of ApoE deficiency shifted from stimulating neural proliferation to reducing it, while we found reduced Ki67expressing cells at 10 weeks of age, a time when ApoE deficiency is correlated with a reduction in proliferation.

The link between the presence of the ApoE4 isoform and the development of Alzheimer's disease and other cognitive deficits in humans suggests that this isoform impairs normal hippocampal functioning. Despite this correlation, the current study did not find any differences in neuronal specification, hippocampal cell proliferation, proliferation of type $1 \mathrm{NSPCs}$, or the total number of type 1 NSPCs in the dentate gyrus of 10-week-old mice expressing ApoE4 when compared with those expressing the human wild-type ApoE3. Previous studies present conflicting evidence regarding the role of the ApoE4 iso- form in hippocampal neurogenesis. Li et al. (2009) found that the expression of ApoE4 does not affect the proliferation and number of NSPCs, results that are supported by the present study, while increasing overall hippocampal cellular proliferation and inhibiting the maturation of newborn neurons in 6- to 7-month-old mice. However, more recently, it has been reported (Koutseff et al., 2014) that ApoE4 leads to decreased levels of hippocampal cell proliferation in 10- to 12-week-old mice compared with ApoE3 mice, a difference that is attenuated as mice mature. Together, these findings suggest that the effect of ApoE4 on neurogenesis is likely age dependent.

The difference in Ki67-expressing cells between ApoEdeficient mice and ApoE4 mice was also interesting. In the present study ApoE-deficient mice were compared with WT mice to reveal the consequences of absent ApoE, while ApoE4 mice were compared with ApoE3 mice to investigate the effects resulting from different human alleles. Therefore, it is not surprising that we observed a significant decrease of Ki67-expressing cells in ApoEdeficient mice at the chosen time but not in ApoE4 mice. By using another marker, Sox2, to investigate early neural progenitors in ApoE4 and ApoE3 targeted replacement mice, no significant differences were reported ( $\mathrm{Li}$ et al., 2009). Therefore, while there may be a nominal effect of ApoE4 on neural proliferation, it does not phenocopy the ApoE-deficient state, suggesting more of a hypomorphic role consistent with what we observed in the dendritic and spine analyses.

During normal neurogenesis, adult-born granule cells project their dendrites toward the molecular layer and form synapses on the medial and lateral perforant pathways (Amaral et al., 2007). Morphologic analysis of mature granule cell dendritic arborizations revealed a wild-type phenotype for wild-type and ApoE3-expressing mice and an abnormal phenotype for ApoE-deficient and ApoE4expressing mice. ApoE4- and ApoE-deficient dentate gyrus adult-born neurons have decreased complexity in their dendritic trees, as well as a decreased total dendritic length. A similar observation was reported in older mice where hippocampal granule cells in ApoE4 mice exhibit less complex dendritic arborizations, which was not observed in ApoE-deficient mice (Li et al., 2009). The present study also highlights that ApoE-deficient and ApoE4 mice have a higher proportion of neurons with less complexity while having a reduced proportion of normal/complex neurons.

The role of cholesterol in neurite outgrowth has been established by culturing retinal ganglion cells with astrocyte-conditioned media (Pfrieger, 2002), and it was subsequently established that astrocyte-secreted cholesterol induced the formation of synapses in vitro (Goritz et al., 2005). Because the transportation of cholesterol 
A

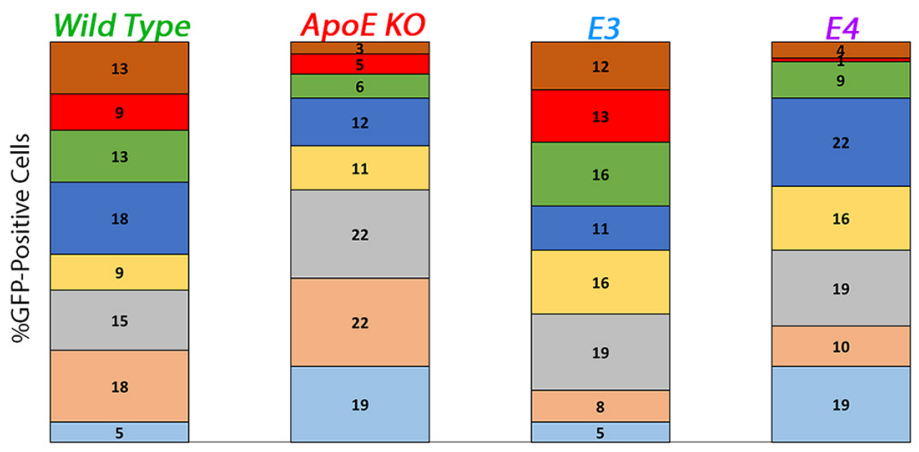

B
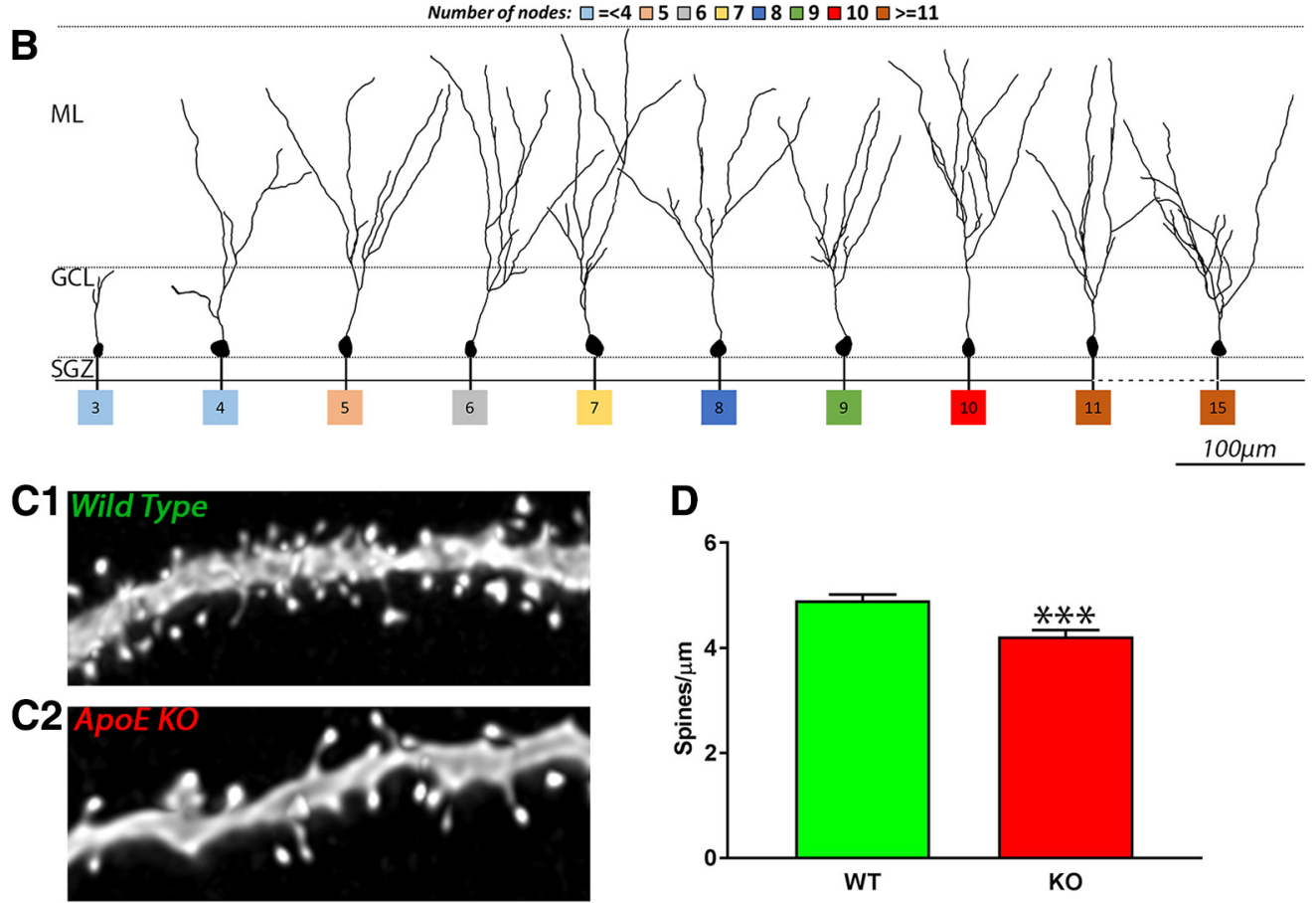

D
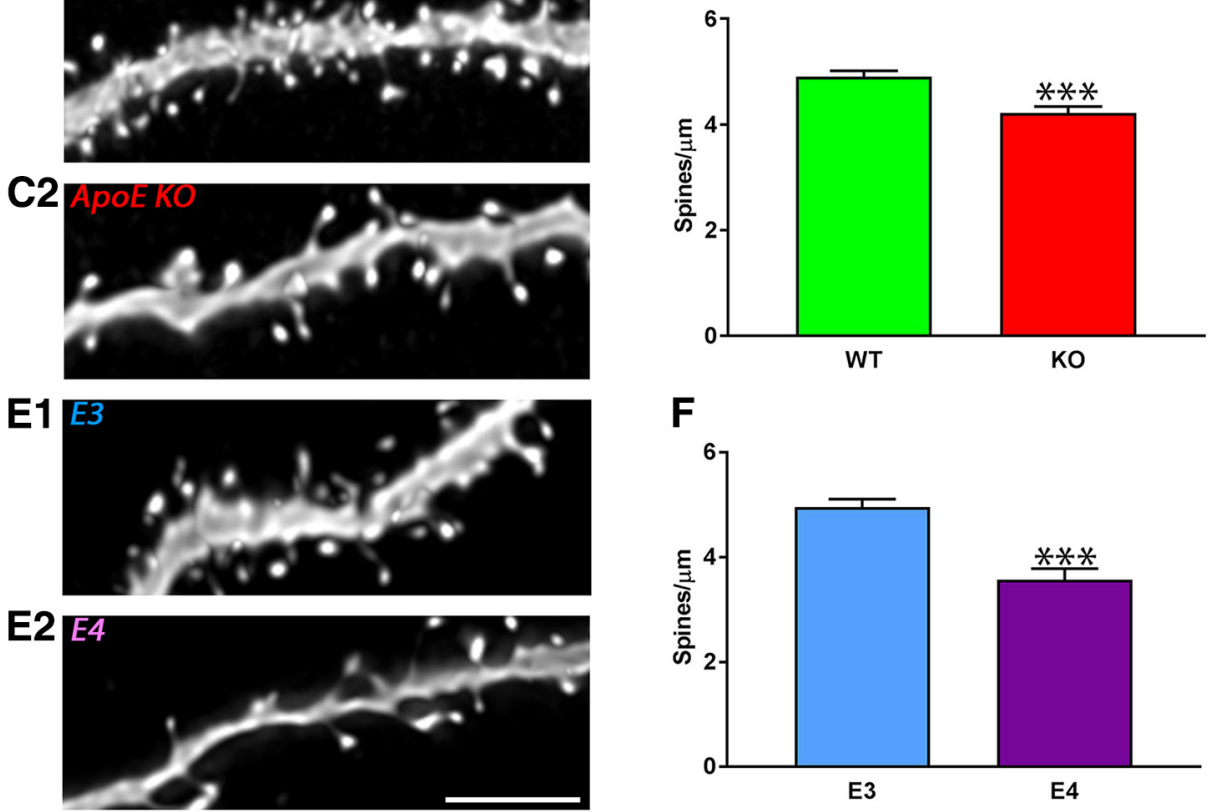

Figure 5. Decrease in the proportion of complex and increase of less complex adult-born granule cells in ApoE-deficient and ApoE4 mice. $\boldsymbol{A}$, Each population of 3D-reconstructed neurons was divided as a function of the number of nodes $(\leq 4, \geq 5,6$. . .10, 11 nodes or more) and expressed as a percentage of the sample function of the different genotypes: wild type (4 mice, 78 neurons); ApoE-deficient (7 mice, 73 neurons); ApoE3 (6 mice, 75 neurons); and ApoE4 mice (4 mice, 69 neurons). $\boldsymbol{B}$, Representative pictures of 3D-reconstructed wild-type adult-born granule cells 4 weeks after GFP retroviral infection at different levels of dendritic complexity. C1, C2, E1, E2, Representative pictures of dendritic fragments from wild-type (C1), ApoE-deficient (C2), ApoE3 (E1), and ApoE4 (E2) mature adult-born granule cells. $\boldsymbol{D}, \boldsymbol{F}$, Spine density quantification in ApoE-deficient (51 fragments from five mice) compared with wild-type (58 fragments from four mice) mice expressed as spines $/ \mu \mathrm{m} \pm \mathrm{SEM}$, and in ApoE4 (46 fragments from four mice) compared with ApoE3 mice (44 fragments from four mice). Independent $t$ test: $* * * * p<0.0001$. Scale bar, E2, $5 \mu \mathrm{m}$.

from astrocytes to neurons is mediated by ApoEcontaining lipoproteins, it has been proposed that cholesterol affects neurite formation and synaptogenesis in an ApoE-dependent manner mediated via the low-density lipoprotein receptor (Mamotte et al., 1999). Therefore, it is not surprising that deficits in ApoE could result in impaired neurite formation. Several studies have demonstrated that human ApoE4 inhibits the neurite outgrowth of cultured 
cortical neurons while ApoE3 promotes it (Nathan et al., 2002). Here we observe that both the deficiency of rodent ApoE and the presence of human ApoE4 resulted in less complexity in newborn neurons, results that are consistent with other findings.

Fewer branches of dendritic trees and lower spine density in newborn neurons in the absence of $A p o E$ and the presence of human ApoE4 were observed when compared with WT and ApoE3 mice. With the narrower angle, fewer dendritic branches, and lower spine density in newborn neurons in the absence of ApoE, neurons born in an ApoE-deficient state are able to receive inputs from both the lateral and medial entorhinal cortex with fewer crossings. However, in the presence of ApoE4, where we observe fewer branches, shorter dendritic length, and lower spine density, the input from the lateral entorhinal cortex may be impaired. Hence, it is not surprising that impairments in performance in hippocampus-dependent behavioral tasks in mice deficient in ApoE or ones carrying the human ApoE4 are observed. Several studies have demonstrated that the presence of human ApoE4 or the absence of rodent ApoE impaired odor memory, Morris water maze task, and contextual fear-conditioning tasks (Masliah et al., 1997; Grootendorst et al., 2001; Peister et al., 2006; Rodriguez et al., 2013; Salomon-Zimri et al., 2015; Peng et al., 2017; East et al., 2018). Further studies are required to determine the electrophysiology of newborn neurons under such genetic backgrounds to see whether they mimic the deficits in behavioral performance.

Dendritic spines are known to be highly variable in size and shape, dynamic, and plastic in vivo (Izeddin et al., 2011), and both their morphology and density are abnormal in several neuropsychiatric disorders such as schizophrenia and Alzheimer's disease (Penzes et al., 2011). The present study revealed a significant decrease in the spine density of both ApoE-deficient and ApoE4 mice, which is consistent with previous work that demonstrated decreased spine density in ApoE-deficient mice at 12 months when compared with wild-type and ApoE3 mice. This decrease was also seen when comparing human $A D$ and age-matched normal control samples carrying one or two copies of the E4 allele compared with samples from individuals without the E4 allele (Ji et al., 2003). Interestingly, the expression of ApoE diminishes when developing into mature neurons from active neural progenitors. The observed deficits in complexity and dendritogenesis in ApoE4 and ApoE-deficient mice might be derived from astrocytic ApoE, as many studies have implicated an astrocytic requirement for proper dendritogenesis (Jain et al., 2013; Perez-Alvarez et al., 2014; Sultan et al., 2015).

Together, our findings reveal a central role of ApoE in dentate gyrus neurogenesis, specifically in the maintenance of the NSPC pool and in the activation of quiescent type 1 cells. Most intriguingly, we observed changes in the morphologic maturation of granule cells as well as their dendritic spine development at a time when they no longer express ApoE, although the proximity of ApoEexpressing astrocytes suggests that, unlike what has been shown with the regulation of NSPC proliferation
(Yang et al., 2011), the effect of ApoE on dendritogenesis appears to occur non-cell autonomously, although the mechanism of how this happens remains entirely unknown. We have also shown that human ApoE4 has an effect that is similar to ApoE deficiency in impairing the dendritic maturation of adult-born granule cells, which suggests important consequences at the neuronal network level. We observed these ultrastructural changes in young (6-week-old) mice, far earlier than the onset ApoE4-associated late-onset Alzheimer's disease. The observations here may represent early lesions that if related to the development of subsequent neurologic disease, may be due to the cumulative effects of these changes over time. Because hippocampal circuitry and neurogenesis play crucial roles in learning and memory, those morphologic aberrances may help to explain, at least in part, the emergence of cognitive decline in humans carrying the $\mathrm{E} 4$ allele and provide a link between the E4 allele and hippocampal-related neurologic diseases.

\section{References}

Allen KM, Fung SJ, Weickert CS (2016) Cell proliferation is reduced in the hippocampus in schizophrenia. Aust N Z J Psychiatry 50: 473-480. CrossRef Medline

Amaral DG, Scharfman HE, Lavenex P (2007) The dentate gyrus: fundamental neuroanatomical organization (dentate gyrus for dummies). Prog Brain Res 163:3-22. CrossRef Medline

Bell RD, Winkler EA, Singh I, Sagare AP, Deane R, Wu Z, Holtzman DM, Betsholtz C, Armulik A, Sallstrom J, Berk BC, Zlokovic BV (2012) Apolipoprotein E controls cerebrovascular integrity via cyclophilin A. Nature 485:512-516. CrossRef Medline

Cho K-O, Lybrand ZR, Ito N, Brulet R, Tafacory F, Zhang L, Good L, Ure K, Kernie SG, Birnbaum SG, Scharfman HE, Eisch AJ, Hsieh J (2015) Aberrant hippocampal neurogenesis contributes to epilepsy and associated cognitive decline. Nat Commun 6:6606. CrossRef Medline

Dickstein DL, Dickstein DR, Janssen WGM, Hof PR, Glaser JR, Rodriguez A, O'Connor N, Angstman P, Tappan SJ (2016) Automatic dendritic spine quantification from confocal data with $\mathrm{Neu}-$ rolucida 360. Curr Protoc Neurosci 77:1.27.1-1.27.21. CrossRef

Dumitriu D, Rodriguez A, Morrison JH (2011) High-throughput, detailed, cell-specific neuroanatomy of dendritic spines using microinjection and confocal microscopy. Nat Protoc 6:1391-1411. CrossRef Medline

East BS, Fleming G, Peng K, Olofsson JK, Levy E, Mathews PM, Wilson DA (2018) Human apolipoprotein E genotype differentially affects olfactory behavior and sensory physiology in mice. Neuroscience 380:103-110. CrossRef Medline

Gilley JA, Yang C-P, Kernie SG (2011) Developmental profiling of postnatal dentate gyrus progenitors provides evidence for dynamic cell-autonomous regulation. Hippocampus 21:33-47. CrossRef Medline

Goritz C, Mauch DH, Pfrieger FW (2005) Multiple mechanisms mediate cholesterol-induced synaptogenesis in a CNS neuron. Mol Cell Neurosci 29:190-201. CrossRef

Grootendorst J, de Kloet ER, Dalm S, Oitzl MS (2001) Reversal of cognitive deficit of apolipoprotein $E$ knockout mice after repeated exposure to a common environmental experience. Neuroscience 108:237-247. Medline

Hartman RE, Wozniak DF, Nardi A, Olney JW, Sartorius L, Holtzman DM (2001) Behavioral phenotyping of GFAP-apoE3 and -apoE4 transgenic mice: apoE4 mice show profound working memory impairments in the absence of Alzheimer's-like neuropathology. Exp Neurol 170:326-344. CrossRef 
Hauser PS, Narayanaswami V, Ryan RO (2011) Apolipoprotein E: from lipid transport to neurobiology. Prog Lipid Res 50:62-74. CrossRef Medline

Hollands C, Bartolotti N, Lazarov O (2016) Alzheimer's disease and hippocampal adult neurogenesis; exploring shared mechanisms. Front Neurosci 10:178. CrossRef Medline

Hong S, Washington PM, Kim A, Yang C-P, Yu T-S, Kernie SG (2016) Apolipoprotein $E$ regulates injury-induced activation of hippocampal neural stem and progenitor cells. J Neurotrauma 33:362-374. CrossRef Medline

Izeddin I, Specht CG, Lelek M, Darzacq X, Triller A, Zimmer C, Dahan $M$ (2011) Super-resolution dynamic imaging of dendritic spines using a low-affinity photoconvertible actin probe. PLoS One 6:e15611. CrossRef Medline

Jain S, Yoon SY, Leung L, Knoferle J, Huang Y (2013) Cellular source-specific effects of apolipoprotein (apo) E4 on dendrite arborization and dendritic spine development. PLoS One 8:e59478. CrossRef Medline

Ji Y, Gong Y, Gan W, Beach T, Holtzman DM, Wisniewski T (2003) Apolipoprotein $\mathrm{E}$ isoform-specific regulation of dendritic spine morphology in apolipoprotein E transgenic mice and Alzheimer's disease patients. Neuroscience 122:305-315. Medline

Jiang Q, Lee CYD, Mandrekar S, Wilkinson B, Cramer P, Zelcer N, Mann K, Lamb B, Willson TM, Collins JL, Richardson JC, Smith JD, Comery TA, Riddell D, Holtzman DM, Tontonoz P, Landreth GE (2008) ApoE promotes the proteolytic degradation of Abeta. Neuron 58:681-693. CrossRef

Jordan BD, Relkin NR, Ravdin LD, Jacobs AR, Bennett A, Gandy S (1997) Apolipoprotein E epsilon4 associated with chronic traumatic brain injury in boxing. JAMA 278:136-140. Medline

Kempermann G, Song H, Gage FH (2015) Neurogenesis in the adult hippocampus. Cold Spring Harb Perspect Biol 7:a018812. CrossRef Medline

Koutseff A, Mittelhaeuser C, Essabri K, Auwerx J, Meziane H (2014) Impact of the apolipoprotein $\mathrm{E}$ polymorphism, age and sex on neurogenesis in mice: pathophysiological relevance for Alzheimer's disease?. Brain Res 1542:32-40. CrossRef Medline

Li G, Bien-Ly N, Andrews-Zwilling Y, Xu Q, Bernardo A, Ring K, Halabisky B, Deng C, Mahley RW, Huang Y (2009) GABAergic interneuron dysfunction impairs hippocampal neurogenesis in adult apolipoprotein E4 knockin mice. Cell Stem Cell 5:634-645. CrossRef Medline

Mamotte CD, Sturm M, Foo JI, van Bockxmeer FM, Taylor RR (1999) Comparison of the LDL-receptor binding of VLDL and LDL from apoE4 and apoE3 homozygotes. Am J Physiol 276:E553-E557. Medline

Masliah E, Samuel W, Veinbergs I, Mallory M, Mante M, Saitoh T (1997) Neurodegeneration and cognitive impairment in apoEdeficient mice is ameliorated by infusion of recombinant apoE. Brain Res 751:307-314. Medline

Mauch DH, Nägler K, Schumacher S, Göritz C, Müller EC, Otto A, Pfrieger FW (2001) CNS synaptogenesis promoted by glia-derived cholesterol. Science 294:1354-1357. CrossRef Medline
Merritt VC, Arnett PA (2016) Apolipoprotein E (APOE) $\epsilon 4$ allele is associated with increased symptom reporting following sports concussion. J Int Neuropsychol Soc 22:89-94. CrossRef Medline

Nathan BP, Jiang Y, Wong GK, Shen F, Brewer GJ, Struble RG (2002) Apolipoprotein E4 inhibits, and apolipoprotein E3 promotes neurite outgrowth in cultured adult mouse cortical neurons through the low-density lipoprotein receptor-related protein. Brain Res 928:96-105. Medline

Peister A, Zeitouni S, Pfankuch T, Reger RL, Prockop DJ, Raber J (2006) Novel object recognition in Apoe(-/-) mice improved by neonatal implantation of wild-type multipotential stromal cells. Exp Neurol 201:266-269. CrossRef Medline

Peng KY, Mathews PM, Levy E, Wilson DA (2017) Apolipoprotein E4 causes early olfactory network abnormalities and short-term olfactory memory impairments. Neuroscience 343:364-371. CrossRef Medline

Penzes P, Cahill ME, Jones KA, VanLeeuwen J-E, Woolfrey KM (2011) Dendritic spine pathology in neuropsychiatric disorders. Nat Neurosci 14:285-293. CrossRef Medline

Perez-Alvarez A, Navarrete M, Covelo A, Martin ED, Araque A (2014) Structural and functional plasticity of astrocyte processes and dendritic spine interactions. J Neurosci 34:12738-12744. CrossRef Medline

Perry EK, Johnson M, Ekonomou A, Perry RH, Ballard C, Attems J (2012) Neurogenic abnormalities in Alzheimer's disease differ between stages of neurogenesis and are partly related to cholinergic pathology. Neurobiol Dis 47:155-162. CrossRef Medline

Pfrieger FW (2002) Role of glia in synapse development. Curr Opin Neurobiol 12:486-490. Medline

Rodriguez GA, Burns MP, Weeber EJ, Rebeck GW (2013) Young APOE4 targeted replacement mice exhibit poor spatial learning and memory, with reduced dendritic spine density in the medial entorhinal cortex. Learn Mem 20:256-266. CrossRef Medline

Sahay A, Hen R (2007) Adult hippocampal neurogenesis in depression. Nat Neurosci 10:1110-1115. CrossRef Medline

Salomon-Zimri S, Liraz O, Michaelson DM (2015) Behavioral testing affects the phenotypic expression of APOE $\varepsilon 3$ and APOE $\varepsilon 4$ in targeted replacement mice and reduces the differences between them. Alzheimers Dement 1:127-135. CrossRef Medline

Sultan S, Li L, Moss J, Petrelli F, Cassé F, Gebara E, Lopatar J, Pfrieger FW, Bezzi P, Bischofberger J, Toni N (2015) Synaptic integration of adult-born hippocampal neurons is locally controlled by astrocytes. Neuron 88:957-972. CrossRef Medline

Teasdale GM, Nicoll JA, Murray G, Fiddes M (1997) Association of apolipoprotein $\mathrm{E}$ polymorphism with outcome after head injury. Lancet 350:1069-1071. CrossRef Medline

Toni N, Schinder AF (2015) Maturation and functional integration of new granule cells into the adult hippocampus. Cold Spring Harb Perspect Biol 8:a018903. CrossRef Medline

Vance JE, Campenot RB, Vance DE (2000) The synthesis and transport of lipids for axonal growth and nerve regeneration. Biochim Biophys Acta 1486:84-96. Medline

Yang C-P, Gilley JA, Zhang G, Kernie SG (2011) ApoE is required for maintenance of the dentate gyrus neural progenitor pool. Development 138:4351-4362. CrossRef Medline 